\title{
Interacting Fermi gases in disordered one-dimensional lattices
}

\author{
Gao Xianlong, ${ }^{1}$ M. Polini, ${ }^{1}$ B. Tanatar, ${ }^{2}$ and M. P. Tosi ${ }^{1}$ \\ ${ }^{1}$ NEST-CNR-INFM and Scuola Normale Superiore, I-56126 Pisa, Italy \\ ${ }^{2}$ Department of Physics, Bilkent University, Bilkent, 06800 Ankara, Turkey \\ (Received 6 March 2006; revised manuscript received 4 April 2006; published 19 April 2006)
}

\begin{abstract}
Interacting two-component Fermi gases loaded in a one-dimensional (1D) lattice and subject to harmonic trapping exhibit intriguing compound phases in which fluid regions coexist with local Mott-insulator and/or band-insulator regions. Motivated by experiments on cold atoms inside disordered optical lattices, we present a theoretical study of the effects of a random potential on these ground-state phases. Within a densityfunctional scheme we show that disorder has two main effects: (i) it destroys the local insulating regions if it is sufficiently strong compared with the on-site atom-atom repulsion, and (ii) it induces an anomaly in the compressibility at low density from quenching of percolation.
\end{abstract}

DOI: 10.1103/PhysRevB.73.161103

PACS number(s): 71.30. $+\mathrm{h}$, 71.10.Pm, 03.75.Lm, 03.75.Ss

\section{INTRODUCTION}

The interplay between interactions and disorder in quantum many-body systems is an area of long-standing interest. For instance, both long-ranged Coulomb interactions and disorder from various mechanisms are believed to play an important role in the metal-insulator transition (MIT) in the two-dimensional (2D) electron liquid. ${ }^{1}$ Disorder and interactions affect not only transport properties of the $2 \mathrm{D}$ electron liquid, but also thermodynamic quantities such as the compressibility" 2,3 and the spin susceptibility. "Dirty-boson" systems such as liquid ${ }^{4} \mathrm{He}$ absorbed in aerogel, Vycor, or Geltech, ${ }^{5}$ or disordered granular superconductors, ${ }^{6}$ have also been extensively studied.

Cold atom gases are becoming important tools to understand the interplay between single-particle randomness and cooperative effects such as superfluidity and many-body effects induced by interactions. ${ }^{7}$ Atoms trapped in an optical lattice (OL) are particularly suitable candidates for such studies, especially because they allow one to reach the strongly coupling regime through the depression of the kinetic energy associated with well-to-well tunneling. ${ }^{8} \mathrm{~A}{ }^{87} \mathrm{Rb}$ BoseEinstein condensate inside a disordered one-dimensional (1D) OL has been used to study the interplay between repulsive interactions and disorder. ${ }^{9}$ In this work it has also been pointed out that thermodynamic quantities, such as the superfluid density, provide a better indicator of disorderinduced localization than time-of-flight absorption images. The present work has been motivated by the experiments in Refs. 7 and 9. We report a study of the interplay between interactions and randomness in a repulsive two-component Fermi gas trapped in a 1D OL. Two-component Fermi gases have recently been prepared in a quasi-1D geometry, ${ }^{10}$ thus opening the way to experimental studies of $1 \mathrm{D}$ phenomena such as spin-charge separation. ${ }^{11}$

The ground state of an interacting Fermi gas moving under harmonic confinement in a 1D OL shows in the absence of disorder five qualitatively different phases $^{12,13}$ (for a pictorial description see Fig. 1). How does disorder influence these phases and their thermodynamic properties? In the following we provide a quantitative answer to this question. In particular, we demonstrate that the incompressible Mottinsulating regions are very stable against disorder at strong coupling. We also show that the compressibility exhibits a disorder-induced low-density anomaly, similar in some respects to the one which has been found both experimentally ${ }^{2}$ and theoretically ${ }^{3}$ in the $2 \mathrm{D}$ electron liquid close to the onset of the MIT.

\section{THE 1D RANDOM FERMI-HUBBARD MODEL}

We consider a two-component Fermi gas with $N_{\text {f }}$ atoms constrained to move under harmonic confinement of strength $V_{2}$ inside a disordered 1D OL with unit lattice constant and $N_{\mathrm{s}}$ lattice sites $i \in\left[1, N_{\mathrm{s}}\right]$. The system is described by a single-band Hubbard Hamiltonian,

$$
\hat{\mathcal{H}}=\hat{\mathcal{H}}_{0}+\sum_{i} \varepsilon_{i} \hat{n}_{i}
$$

where $\quad \hat{\mathcal{H}}_{0}=-t \Sigma_{i, \sigma}\left(\hat{c}_{i \sigma}^{\dagger} \hat{c}_{i+1 \sigma}+\right.$ H.c. $)+U \sum_{i} \hat{n}_{i \uparrow} \hat{n}_{i \downarrow}+V_{2} \sum_{i}(i$ $\left.-N_{\mathrm{s}} / 2\right)^{2} \hat{n}_{i}$. Here $\sigma=\uparrow, \downarrow$ is a pseudospin-1/2 label for two

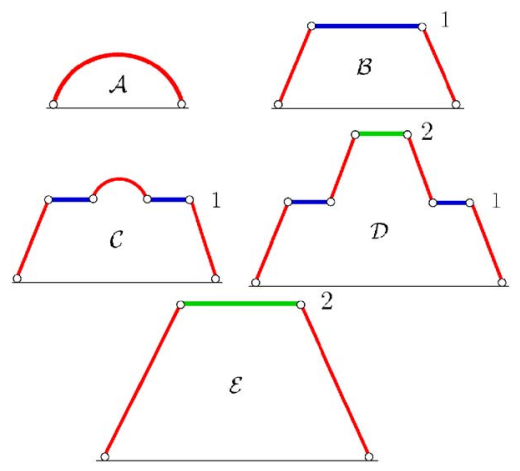

FIG. 1. (Color online) Sketch of the site occupation $n_{i}$ of an interacting Fermi gas in a harmonic trap and a clean 1D lattice. Phase $\mathcal{A}$ is a fluid with $0<n_{i}<2$. In phase $\mathcal{B}$ an incompressible Mott insulator occupies the bulk of the trap with $n_{i}$ locally locked to 1. In phase $\mathcal{C}$ a fluid with $1<n_{i}<2$ is embedded in the Mott plateau. In phase $\mathcal{D}$ a band insulator with $n_{i}$ locally locked to 2 is surrounded by fluid edges and embedded in the Mott plateau. Finally, in phase $\mathcal{E}$ a band insulator in the bulk of the trap coexists with fluid edges. 
internal hyperfine states, $\hat{n}_{i}=\sum_{\sigma} \hat{n}_{i \sigma}=\sum_{\sigma} \hat{c}_{i \sigma}^{\dagger} \hat{c}_{i \sigma}$ is the total site occupation operator. The effect of disorder is simulated by the last term in Eq. (1), where $\varepsilon_{i}$ is randomly chosen at each site from a uniform distribution in the range $[-W / 2, W / 2]$ (Ref. 14).

In the unconfined limit $\left(V_{2}=0\right)$ the Hamiltonian $\hat{\mathcal{H}}$ reduces to the Anderson localization problem ${ }^{15}$ for $U=0$ and to the exactly solvable Lieb-Wu model ${ }^{16}$ for $W=0$. The Lieb-Wu model describes a Luttinger liquid away from half and full filling, a Mott insulator at half filling, and a band insulator at full filling. In the unconfined limit the 2D version of $\hat{\mathcal{H}}$ has been studied in connection with the 2D MIT (Ref. 17). In the clean limit five different phases have been identified ${ }^{12,13}$ (see Fig. 1).

A particular set $\varepsilon_{i}(\alpha)$ of randomly chosen values of $\varepsilon_{i}$, labeled by $\alpha$, is a realization of disorder. Each realization defines an external potential $\mathcal{V}_{i}(\alpha) \equiv V_{2}\left(i-N_{\mathrm{s}} / 2\right)^{2}+\varepsilon_{i}(\alpha)$ which determines, via site-occupation functional theory, ${ }^{18}$ a site occupation $n_{i}(\alpha)=\left\langle\Psi_{\alpha}\left|\hat{n}_{i}\right| \Psi_{\alpha}\right\rangle,\left|\Psi_{\alpha}\right\rangle$ being the ground state (GS) of $\hat{\mathcal{H}}$ for disorder realization $\alpha$. The total energy is a unique functional of $n_{i}(\alpha)$, which can be written as ${ }^{13}$ $\mathcal{E}[n(\alpha)]=\mathcal{F}_{\mathrm{HK}}[n(\alpha)]+\sum_{i} \mathcal{V}_{i}(\alpha) n_{i}(\alpha)$. Here $\mathcal{F}_{\mathrm{HK}}[n(\alpha)]$ is the universal Hohenberg-Kohn (HK) functional. The GS site occupation can be found by solving the Euler-Lagrange equation

$$
\frac{\delta \mathcal{F}_{\mathrm{HK}}[n(\alpha)]}{\delta n_{i}(\alpha)}+\mathcal{V}_{i}(\alpha)=\mu(\alpha),
$$

with the constant $\mu(\alpha)$ being a Lagrange multiplier to enforce particle conservation. A local-density approximation (LDA) will be used below for $\mathcal{F}_{\mathrm{HK}}[n(\alpha)]$.

The site occupation $\mathcal{N}_{i}$ due to $\hat{\mathcal{H}}$ is finally obtained by means of a disorder ensemble average, i.e., $\mathcal{N}_{i}=\left\langle\left\langle n_{i}\right\rangle\right\rangle_{\text {dis }}$ where $\langle\langle\mathcal{O}\rangle\rangle_{\mathrm{dis}}=\lim _{\mathcal{M} \rightarrow \infty}(1 / \mathcal{M}) \sum_{\alpha=1}^{\mathcal{M}} \mathcal{O}(\alpha)$. In practice one can average only over a finite number $\mathcal{M}$ of disorder realizations. Due to our very efficient computational method ${ }^{13}$ we are easily able to average over $\mathcal{M}=10^{4}$ realizations of disorder for every set of parameters $\left\{N_{\mathrm{s}}, N_{\mathrm{f}}, u \equiv U / t\right.$, $\left.V_{2} / t, W / t\right\}$ (Ref. 19). Finally, the global compressibility can be obtained from the stiffness ${ }^{11} \mathcal{S}_{\rho} \equiv\left\langle\left\langle\delta \mu / \delta N_{\mathrm{f}}\right\rangle\right\rangle_{\mathrm{dis}}$.

\section{SITE OCCUPATION AND STIFFNESS ANOMALY IN THE PRESENCE OF DISORDER}

The functional $\mathcal{F}_{\mathrm{HK}}$ is the sum of three terms, $\mathcal{F}_{\mathrm{HK}}[n(\alpha)]=T_{s}[n(\alpha)]+(U / 2) \sum_{i} n_{i}^{2}(\alpha)+\mathcal{E}_{\mathrm{xc}}[n(\alpha)]$. The first term is the noninteracting kinetic-energy functional, which is approximated in this work "à la Thomas-Fermi". ${ }^{13}$ The other terms in $\mathcal{F}_{\mathrm{HK}}$ are the mean-field interaction energy and the exchange-correlation energy functional incorporating manybody effects beyond mean field. This is approximated through an LDA based on the exactly known exchangecorrelation energy of the Lieb-Wu model. ${ }^{13}$ Within this LDA an explicit equation for $n_{i}(\alpha)$ can be derived ${ }^{13}$ from Eq. (2). Its self-consistent solution leads to the site occupation $n_{i}(\alpha)$ and to the chemical potential $\mu(\alpha)$ for a particular disorder
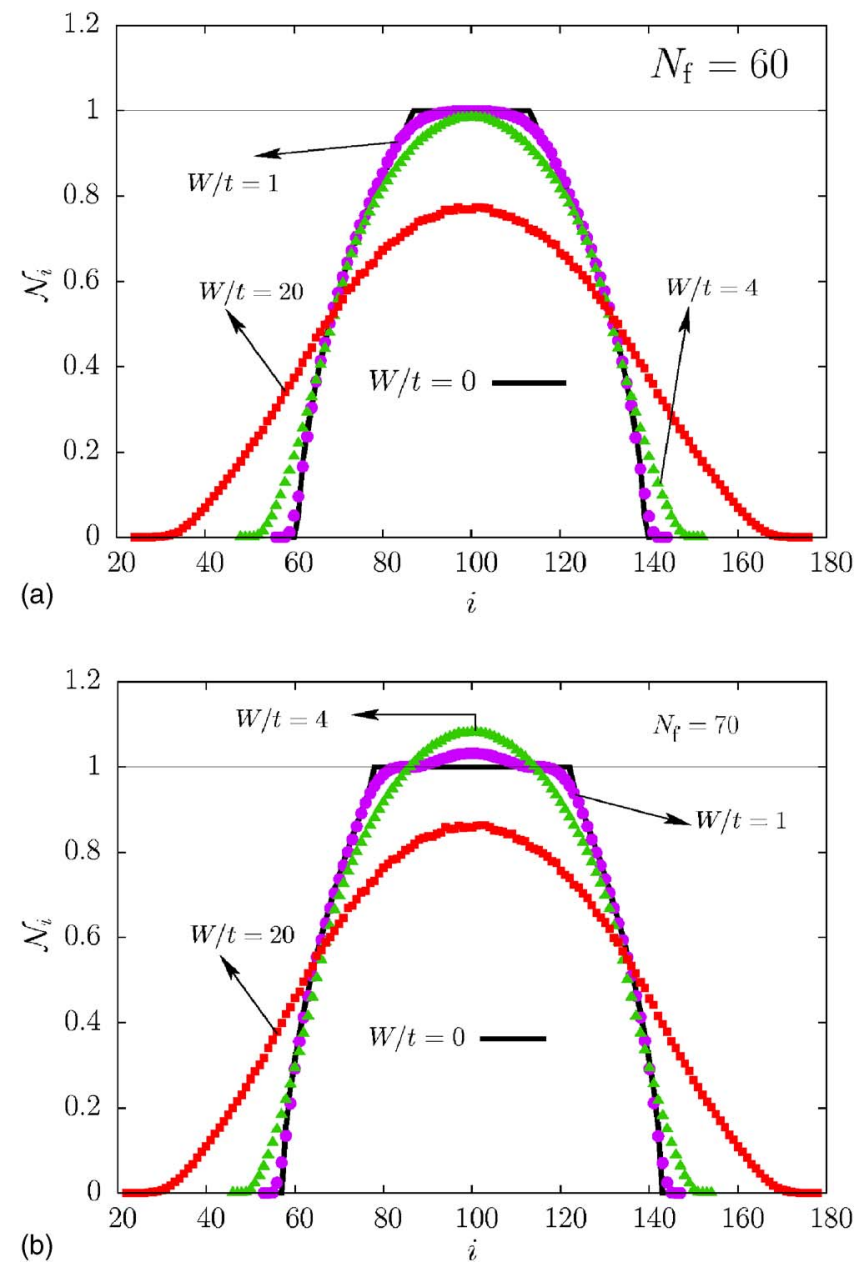

FIG. 2. (Color online) Site occupation $\mathcal{N}_{i}$ as a function of site position $i$ for $u=4$ and $V_{2} / t=2.5 \times 10^{-3}$ in a lattice with $N_{\mathrm{s}}=200$ sites. The number of atoms is $N_{\mathrm{f}}=60$ in the top panel and $N_{\mathrm{f}}=70$ in the bottom panel.

realization, ${ }^{20}$ and hence to the disorder-ensemble averaged quantities.

In Fig. 2 we show the site occupation $\mathcal{N}_{i}$ for a disordered gas with $u=4$, which is in phase $\mathcal{B}$ for $W=0$. The edges of the Mott plateau are the first to be corrupted by the appearence of weak disorder. The stability of the central region of the plateau depends on the number of atoms, if all other parameters are kept fixed. For $N_{\mathrm{f}}=60$ (top panel of Fig. 2) the central region persists over a finite range of disorder. Disorder increases the tunneling through the edges of the trap, making the confinement effectively weaker, and thus leads to broadening of the site occupation. The Mott plateau has disappeared at $W / t=4$, and for $W / t=20$ the siteoccupation profile has a strongly nonparabolic overall shape. For $N_{\mathrm{f}}=70$ instead (bottom panel of Fig. 2), the Mott plateau at the center of the trap is unstable against the formation of a fluid phase with $\mathcal{N}_{i}>1$, and the Mott phase can survive for weak disorder only in an intermediate region between the edge and the center of the trap. In fact, $N_{\mathrm{f}}=70$ is the critical number of atoms at which the phase transition $\mathcal{B} \rightarrow \mathcal{C}$ occurs in the clean limit: a weak disorder potential can shift the transition and induce a fluid region embedded in the Mott 

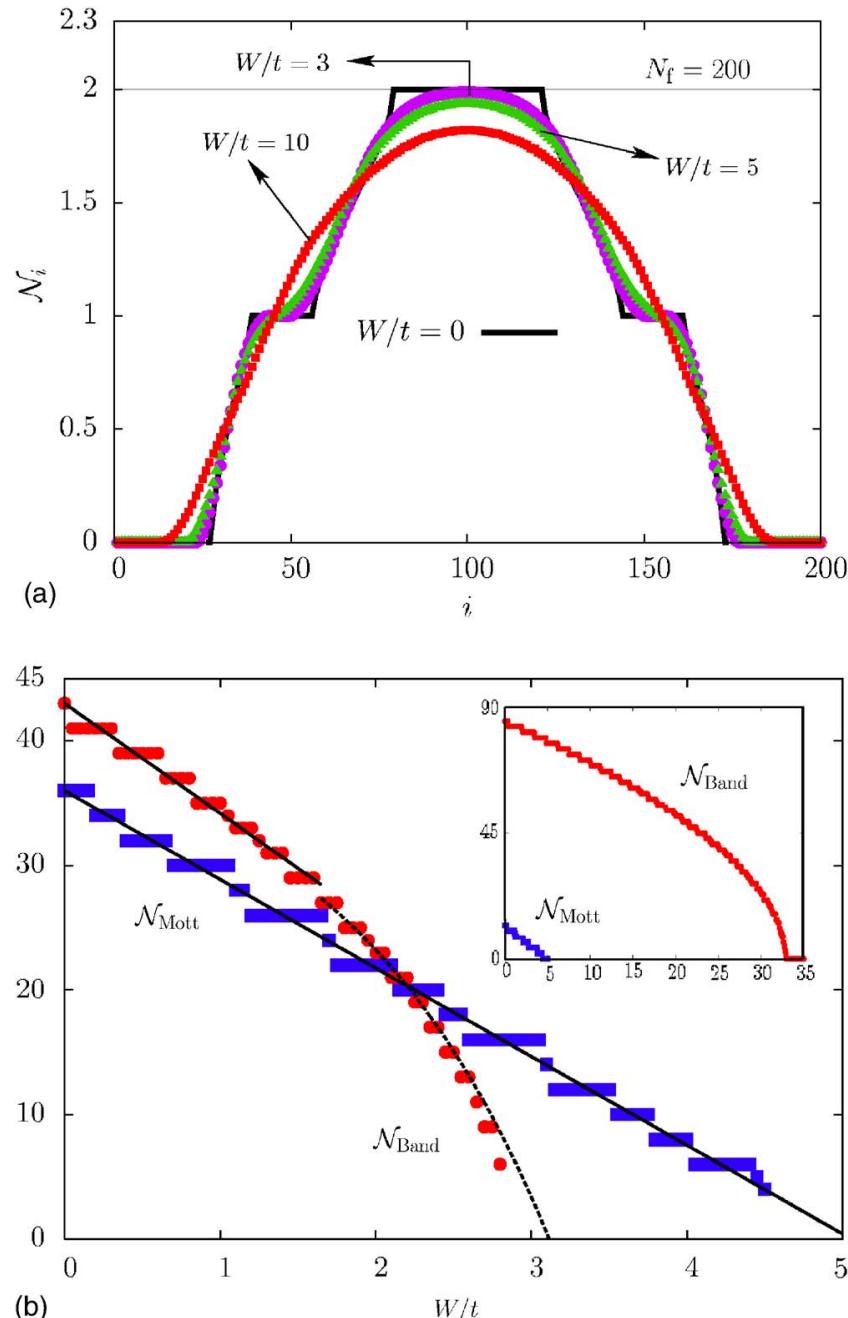

FIG. 3. (Color online) Top panel: Site occupation $\mathcal{N}_{i}$ as a function of $i$ for $N_{\mathrm{f}}=200$ atoms in a lattice with $N_{\mathrm{s}}=200$ sites, in the case $u=8$ and $V_{2} / t=2.5 \times 10^{-3}$. Bottom panel: Number of consecutive sites $\mathcal{N}_{\text {Mott }}\left(\mathcal{N}_{\text {Band }}\right)$ at which $\left|\mathcal{N}_{i}-1\right| \leqslant 10^{-5}\left(\left|\mathcal{N}_{i}-2\right| \leqslant 10^{-5}\right)$, as a function of $W / t$ for the system shown in the top panel. The steps show that each insulating region is stable over a finite range of $W / t$. $\mathcal{N}_{\text {Mott }}$ can be fitted with a linear function over the whole range of $W / t$ [solid line through the squares, showing that $\mathcal{N}_{\text {Mott }}=0$ at $\left.(W / t)_{\mathrm{c} 1} \approx 5\right] . \mathcal{N}_{\text {Band }}$ has a linear behavior up to $W / t \approx 1.8$ and beyond can only be fitted by a nonlinear function [solid and dashed lines through the dots, showing that $\mathcal{N}_{\text {band }}=0$ at $(W / t)_{\mathrm{c} 2} \approx 3$ $\left.<(W / t)_{\mathrm{c} 1}\right]$. In the inset we show $\mathcal{N}_{\text {Mott }}$ and $\mathcal{N}_{\text {Band }}$ for the same system parameters as in the main body of the figure but for $V_{2} / t$ $=8 \times 10^{-3}$. In this case the clean system is close to the $\mathcal{D} \rightarrow \mathcal{E}$ phase transition.

plateau. Eventually, the scenario depicted above for $N_{\mathrm{f}}=60$ is reestablished when $W$ is strong enough.

In the top panel of Fig. 3 we show the site occupation for a disordered gas with $u=8$, which is deep in phase $\mathcal{D}$ for $W=0$. We note that at $W / t=3$ the Mott-insulating regions still exist, while the band-insulating region has been destroyed. This confirms the expectation that a Mott-insulating region, having its origin in exchange-correlation effects, is more stable against disorder than a band-insulating region
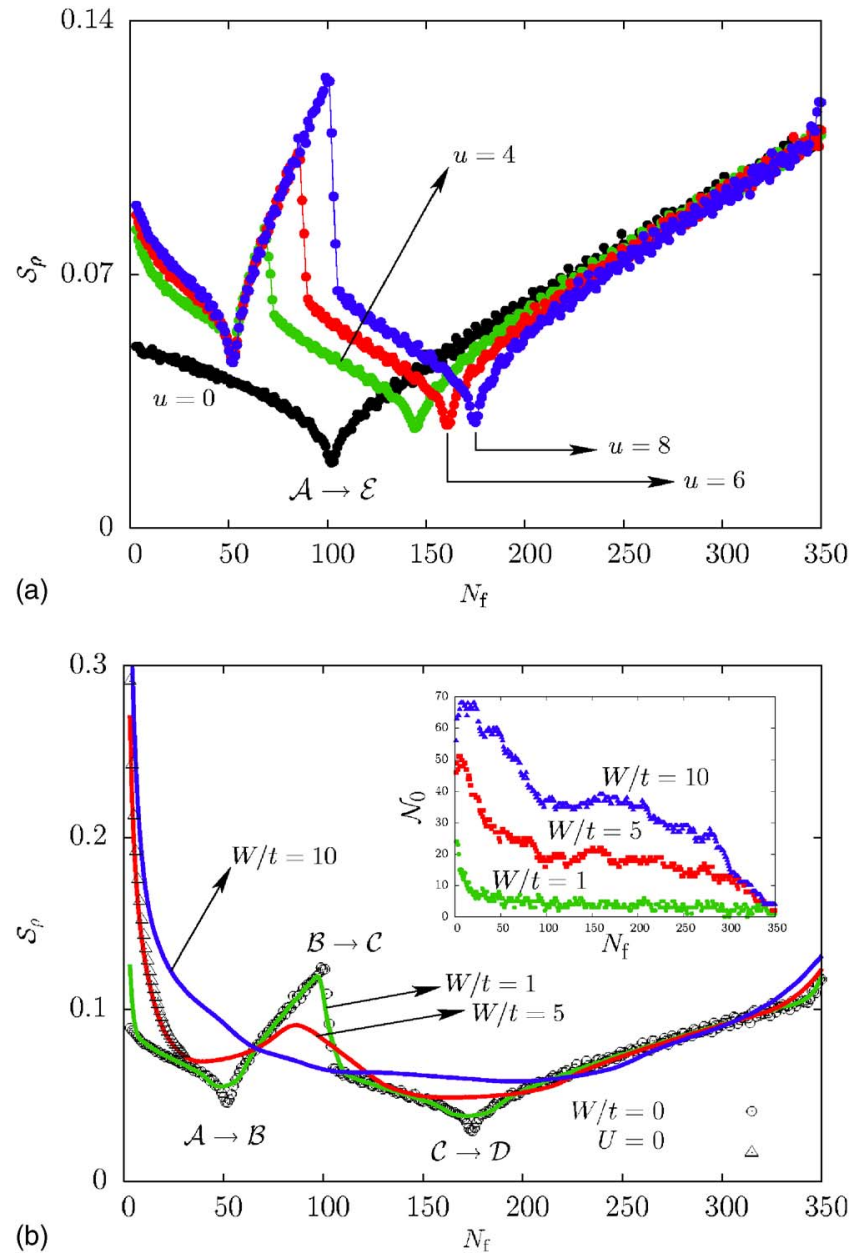

FIG. 4. (Color online) Thermodynamic stiffness $\mathcal{S}_{\rho}$ (in units of $t)$ as a function of $N_{\mathrm{f}}$ for $V_{2} / t=2.5 \times 10^{-3}$ and $N_{\mathrm{s}}=200$ lattice sites. Top panel: results for a clean system at various values of $u$ (in the noninteracting case only the phase transition $\mathcal{A} \rightarrow \mathcal{E}$ can occur). Bottom panel: results for a disordered system at $u=8$ and for $0 \leqslant W / t$ $\leqslant 10$. The black triangles report the low-density stiffness of a noninteracting system at $W / t=10$. The inset shows the number $\mathcal{N}_{0}$ of sites at which $n_{i}(\alpha) \leqslant 10^{-5}$ in a particular realization of disorder, as a function of $N_{\mathrm{f}}$ for $u=8$ and $0 \leqslant W / t \leqslant 10$.

(see the bottom panel of Fig. 3). This statement does not apply when the clean system approaches the transition $\mathcal{D}$ $\rightarrow \mathcal{E}$, simply because the number of sites in the band insulator is much larger than the number of sites in the Mott insulator (see the inset in the bottom panel of Fig. 3).

We turn in Fig. 4 to illustrate the effect of disorder on the stiffness of the Fermi gas. In the top panel we show $\mathcal{S}_{\rho}$ as a function of $N_{\mathrm{f}}$ at different values of $u$ in the absence of disorder. At $u \geqslant 4$ this quantity exhibits three nonanalyticity points associated with the three phase transitions: $\mathcal{A} \rightarrow \mathcal{B}$, $\mathcal{B} \rightarrow \mathcal{C}$, and $\mathcal{C} \rightarrow \mathcal{D}$. The increase in stiffness in phases $\mathcal{B}$ and $\mathcal{D}$ is related to the incompressible nature of the insulating regions present in these two phases. In the bottom panel of Fig. 4 we show the same disorder-averaged quantity for a Fermi gas with $u=8$. We see that the disorder has two main effects. It not only leads to smoothing of the nonanalytic behaviors found in the clean limit, but also induces a strong stiffening at low density. The latter is an "anomalous" behav- 
ior compared to that found in the clean limit. In fact, for finite $W$ the stiffening appears to grow unbounded at very low density (see bottom panel of Fig. 4), following the power law $\mathcal{S}_{\rho} \propto\left(N_{\mathrm{f}}\right)^{-\nu}$ with an exponent $\nu \approx 0.6$. The value of the exponent is essentially independent of the parameters $u$ and $W / t$, but depends on the confinement: for example, we find $\nu \approx 0.4$ for an open lattice with $V_{2}=0$. At high density $\mathcal{S}_{\rho}$ appears instead to be essentially unaffected by disorder.

The low-density behavior of $\mathcal{S}_{\rho}$ is reminiscent of what has been found in Refs. 2 and 3 for a 2 D electron liquid. Following Ref. 3 we explain the origin of the anomaly using the concept of density percolation. As $N_{\mathrm{f}}$ decreases the highdensity regions tend to become disconnected, since the atoms tend to occupy just the deepest valleys in the disorder land- scape. At given $u$ and low $N_{\mathrm{f}}$, the system thus stiffens as the disorder grows (see the bottom panel in Fig. 4). For a given realization of disorder, the number $\mathcal{N}_{0}$ of essentially empty sites increases with $W / t$, as it is shown in the inset. ${ }^{21}$

In summary, we have shown how disorder affects the quantum phases of interacting Fermi gases moving under harmonic confinement in 1D lattices. In particular we have seen that Mott-insulating regions are quite stable against uniformly distributed uncorrelated disorder and that the disorder induces an anomalous increase of the stiffness at low density from quenching of percolation.

This work was partially supported by an Advance Research Initiative of S.N.S. and by TUBITAK and TUBA.
${ }^{1}$ See, e.g., E. Abrahams, S. V. Kravchenko, and M. P. Sarachik, Rev. Mod. Phys. 73, 251 (2001); B. L. Altshuler, D. L. Maslov, and V. M. Pudalov, Physica E (Amsterdam) 9, 209 (2001).

${ }^{2}$ S. Ilani, A. Yacoby, D. Mahalu, and H. Shtrikman, Phys. Rev. Lett. 84, 3133 (2000); S. C. Dultz and H. W. Jiang, ibid. 84, 4689 (2000).

${ }^{3}$ J. Shi and X. C. Xie, Phys. Rev. Lett. 88, 086401 (2002).

${ }^{4}$ A. A. Shashkin, S. Anissimova, M. R. Sakr, S. V. Kravchenko, V. T. Dolgopolov, and T. M. Klapwijk, Phys. Rev. Lett. 96, 036403 (2006).

${ }^{5}$ See, e.g., J. D. Reppy, J. Low Temp. Phys. 87, 205 (1992).

${ }^{6}$ R. Fazio and H. van der Zant, Phys. Rep. 355, 235 (2001).

${ }^{7}$ J. E. Lye, L. Fallani, M. Modugno, D. S. Wiersma, C. Fort, and M. Inguscio, Phys. Rev. Lett. 95, 070401 (2005); D. Clément, A. F. Varón, M. Hugbart, J. A. Retter, P. Bouyer, L. SanchezPalencia, D. M. Gangardt, G. V. Shlyapnikov, and A. Aspect, ibid. 95, 170409 (2005); C. Fort, L. Fallani, V. Guarrera, J. E. Lye, M. Modugno, D. S. Wiersma, and M. Inguscio, ibid. 95, 170410 (2005); V. Ahufinger, L. Sanchez-Palencia, A. Kantian, A. Sanpera, and M. Lewenstein, Phys. Rev. A 72, 063616 (2005).

${ }^{8}$ J. I. Cirac and P. Zoller, Science 301, 176 (2003).

${ }^{9}$ T. Schulte, S. Drenkelforth, J. Kruse, W. Ertmer, J. Arlt, K. Sacha, J. Zakrzewski, and M. Lewenstein, Phys. Rev. Lett. 95, 170411 (2005).

${ }^{10}$ H. Moritz, T. Stöferle, K. Günter, M. Köhl, and T. Esslinger, Phys. Rev. Lett. 94, 210401 (2005).

${ }^{11}$ T. Giamarchi, Quantum Physics in One Dimension (Clarendon, Oxford, 2004).

${ }^{12}$ M. Rigol, A. Muramatsu, G. G. Batrouni, and R. T. Scalettar, Phys. Rev. Lett. 91, 130403 (2003); M. Rigol and A. Muramatsu, Phys. Rev. A 69, 053612 (2004); X.-J. Liu, P. D. Drummond, and H. Hu, Phys. Rev. Lett. 94, 136406 (2005); V. L. Campo, Jr. and K. Capelle, Phys. Rev. A 72, 061602(R) (2005).

${ }^{13}$ Gao Xianlong, M. Polini, M. P. Tosi, V. L. Campo, Jr., K. Capelle, and M. Rigol, cond-mat/0512184, Phys. Rev. B (to be published).
${ }^{14}$ Other disorder models can be chosen, representing, e.g., smooth correlated noise. A comparative study of different disorder models will be given elsewhere.

${ }^{15}$ P. W. Anderson, Phys. Rev. 109, 1492 (1958).

${ }^{16}$ E. H. Lieb and F. Y. Wu, Phys. Rev. Lett. 20, 1445 (1968).

${ }^{17}$ See, e.g., D. Heidarian and N. Trivedi, Phys. Rev. Lett. 93, 126401 (2004).

${ }^{18}$ K. Schönhammer, O. Gunnarsson, and R. M. Noack, Phys. Rev. B 52, 2504 (1995); N. A. Lima, M. F. Silva, L. N. Oliveira, and K. Capelle, Phys. Rev. Lett. 90, 146402 (2003).

${ }^{19}$ Averaging over such large numbers of realizations becomes necessary as the strength of disorder increases and we have checked that the density profiles that we report below are stable against further increases of $\mathcal{M}$.

${ }^{20}$ In the clean limit this LDA scheme has been shown to be reliable through extensive comparisons with accurate quantum Monte Carlo calculations (Ref. 13). LDA-based density-functional schemes for disordered systems have been successfully employed to study, e.g., the low-density compressibility anomaly in the 2D MIT (see Ref. 3) and the statistical properties of 2D disordered quantum dots [see, e.g., E. Räsänen and M. Aichinger, Phys. Rev. B 72, 045352 (2005), and references therein to earlier work].

${ }^{21}$ There is, however, an important conceptual difference between the present Fermi gas and the 2D electron liquid. In the latter the density is also an inverse measure of the coupling strength: the stiffness anomaly at low density occurs in the strongly correlated regime. In the present case the atom number $N_{\mathrm{f}}$ and the interaction parameter $U$ are instead independent parameters. The anomaly that we observe occurs also in the noninteracting limit (see the bottom panel of Fig. 4), demonstrating the crucial role of the quenching of percolation in originating the anomaly. The interatomic repulsions enhance the stiffness at low density in the disordered case just as they do in the clean case, in accordance with the intuitive expectation that a repulsive system is less compressible. 